\title{
Systematic Reduction of the Detailed Kinetic Mechanism for the Combustion of $n$-Butane
}

\author{
Chunhui Liu, ${ }^{1,2}$ Zhengxing Zuo, $^{1}$ and Huihua Feng ${ }^{1}$ \\ ${ }^{1}$ School of Mechanical Engineering, Beijing Institute of Technology, Beijing 100081, China \\ ${ }^{2}$ School of Automobile and Transportation, Tianjin University of Technology and Education, Tianjin 300222, China
}

Correspondence should be addressed to Zhengxing Zuo; zxzuo@bit.edu.cn

Received 14 January 2016; Revised 9 March 2016; Accepted 20 March 2016

Academic Editor: Mark Hoffmann

Copyright (C) 2016 Chunhui Liu et al. This is an open access article distributed under the Creative Commons Attribution License, which permits unrestricted use, distribution, and reproduction in any medium, provided the original work is properly cited.

A systematic approach for mechanism reduction was demonstrated to generate a skeletal and reduced mechanism for the oxidation of $n$-butane. First, a skeletal mechanism, including 89 species and 440 elementary reactions, was derived from a 230 -species detailed mechanism using path flux analysis (PFA). Then, the unimportant reactions were eliminated using the importance index defined in computational singular perturbation (CSP), resulting in a skeletal mechanism consisting of 89 species and 298 elementary reactions. Finally, 20 global quasi-steady-state species were identified using a CSP-based time-scale analysis, leading to a 69-species reduced mechanism. Validation of the 89-species skeletal and 69-species reduced mechanisms showed good agreement with the detailed mechanism for both the ignition delay time and the distribution of species concentration over a wide range of simulation conditions.

\section{Introduction}

Microburners are emerging as a powerful tool to convert available energy into usable forms in small-scale applications [1-4]. $n$-Butane, one of the liquid hydrocarbons, is widely used in microcombustion because of its easy storage and transport. However, its ignition and combustion stability remain a challenge [5]. Understanding the combustion chemistry of $n$-butane is the key to improving the system combustion efficiency and optimizing the design. $n$-Butane is a simple hydrocarbon fuel that exhibits similar combustion characteristics to the more complex hydrocarbon fuels, such as negative temperature coefficient regions (NTC) and two-stage autoignition. However, the detailed mechanism for $n$-butane is highly complex.

Kojima [6] reported a detailed, low temperature, chemical kinetic mechanism of $n$-butane autoignition, consisting of 141 species and 461 reactions, and validated the mechanism against a rapid compression experiment. Warth et al. [7] generated a mechanism including 168 species and 797 reactions for $n$-butane oxidation and validated this mechanism by modeling $n$-butane oxidation at low temperature between 554 and $737 \mathrm{~K}$ in the NTC region and at $937 \mathrm{~K}$. Strelkova et al. [8] developed a skeletal mechanism, including 54 species and
94 reactions, for low temperature $(500-800 \mathrm{~K})$ ignition of $n$-butane. The recently published detailed mechanism from Healy et al. [9] for $n$-butane, comprising 230 species and 1328 reactions, was validated by an experiment both in a rapid compression machine and in a shock tube for a wider range of equivalence ratios (0.3-2), pressures (1-45 atm), and temperatures (690-1430 K).

The detailed chemical kinetic mechanism prevents the computation of complex combustion phenomena, such as two- and three-dimensional combustion, because of the large size and chemical stiffness. Therefore, it is necessary to reduce the detailed mechanism to smaller sizes with less stiffness.

The detailed mechanism reduction can be conducted at two levels. The first level is skeletal reduction using methods such as directed relation graph (DRG) [10], DRG with error propagation (DRGEP) [11], path flux analysis (PFA) [12], revised DRG (DRGMAX) [13], automatic mechanism reduction program ReaxRed (http://ccg.scu.edu.cn/), and computational singular perturbation (CSP) [14-17] to eliminate the unimportant reactions and species. The second level of reduction is global reduction using methods including computational singular perturbation (CSP) [18], quasi-steady-state approximation (QSSA) [19], and intrinsic low dimensional 
manifold (ILDM) [20] to consider the impact of the timescales on the whole reaction system.

In the present study, we generated the smallest skeletal mechanism consisting of 89 species and 440 elementary reactions from Healy's detailed mechanism by comparing four different reduction methods (DRG, DRGEP, DRGMAX, and PFA). Then, the skeletal mechanism was reduced further by the computational singular perturbation (CSP) method to identify and eliminate only the elementary reactions. The final skeletal mechanism consists of 89 species and 298 elementary reactions. We further applied CSP and the QSS assumption for the final skeletal mechanism to generate a reduced mechanism with 69 species.

\section{Reduction Methodologies}

2.1. DRG. The DRG method is an efficient method for skeletal mechanism reduction. In the DRG method, $r_{A B}$, normalized contribution of species $B$ to the production rate of species $A$, is defined as

$$
r_{A B}=\frac{\sum_{i=1, I}\left|v_{A, i} \omega_{i} \delta_{B}^{i}\right|}{\sum_{i=1, I}\left|v_{A, i} \omega_{i}\right|} .
$$

Here, $I$ is the total number of elementary reactions, $v_{A, i}$ is the stoichiometric coefficient of species $A$ in the $i$ th elementary reaction, and $\omega_{i}$ is the net reaction rate of the $i$ th elementary reaction. For $\delta_{B}^{i}$, if $i$ th elementary reaction involves species $B$, $\delta_{B}^{i}=1$; otherwise, $\delta_{B}^{i}=0$.

$r_{A B}$ indicates the dependence of species $B$ on species $A$. If $r_{A B}<\xi$ (threshold), the relation between $B$ and $A$ is considered to be negligible. On the other hand, species $B$ is selected when $r_{A B}>\xi$.

2.2. DRGEP. For the DRGEP method, $r_{A B}$ is defined as

$$
r_{A B}=\frac{\sum_{i=1, I}\left|v_{A, i} \omega_{i} \delta_{B}^{i}\right|}{\max \left(P_{A}, C_{A}\right)},
$$

where

$$
\begin{aligned}
P_{A} & =\sum_{i=1}^{I} \max \left(0, v_{A, i} \omega_{i}\right), \\
C_{A} & =\sum_{i=1}^{I} \max \left(0,-v_{A, i} \omega_{i}\right) .
\end{aligned}
$$

All forward and backward rates must be considered as a single reaction when using the method. As in the DRG method, if $r_{A B}<\xi$, the relation between $B$ and $A$ is considered to be negligible. On the other hand, species $B$ is selected when $r_{A B}>\xi$.

2.3. DRGMAX. For the revised DRG method, $r_{A B}$ is defined as

$$
r_{A B}=\frac{\max _{i}\left(v_{A, i} \omega_{i} \delta_{B}^{i}\right)}{\max _{i}\left(v_{A, i} \omega_{i}\right)}
$$

We can more effectively handle large isomer groups in the detailed mechanism using this method. As in the DRG method, if $r_{A B}<\xi$, the relation between $B$ and $A$ is considered to be negligible. On the other hand, species $B$ is selected when $r_{A B}>\xi$.

2.4. PFA. For the PFA method, $r_{A B}$ of the second generation is defined as

$$
r_{A B}=r_{A B}^{1 \text { st-pro }}+r_{A B}^{1 \text { st-con }}+r_{A B}^{2 \text { nd-pro }}+r_{A B}^{2 \text { nd-con }},
$$

where

$$
\begin{aligned}
& r_{A B}^{\text {1st-pro }} \\
& =\frac{\sum_{i=1, I} \max \left(v_{A, i} \omega_{i} \delta_{B}^{i}, 0\right)}{\max \left(\sum_{i=1, I} \max \left(v_{A, i} \omega_{i}, 0\right), \sum_{i=1, I} \max \left(-v_{A, i} \omega_{i}, 0\right)\right)}, \\
& r_{A B}^{1 \text { st-con }} \\
& =\frac{\sum_{i=1, I} \max \left(-v_{A, i} \omega_{i} \delta_{B}^{i}, 0\right)}{\max \left(\sum_{i=1, I} \max \left(v_{A, i} \omega_{i}, 0\right), \sum_{i=1, I} \max \left(-v_{A, i} \omega_{i}, 0\right)\right)}, \\
& r_{A B}^{2 \text { nd-pro }}=\sum_{M_{i} \neq A, B} r_{A M_{i}}^{1 \text { st-pro }} r_{M_{i} B}^{1 s t-p r o}, \\
& r_{A B}^{2 \text { nd-con }}=\sum_{M_{i} \neq A, B} r_{A M_{i}}^{1 \text { st-con }} r_{M_{i} B}^{1 \text { st-con }} .
\end{aligned}
$$

The production and consumption fluxes are used to identify the important reaction pathways in this method. As in the DRG method, if $r_{A B}<\xi$, the relation between $B$ and $A$ is considered to be negligible. On the other hand, species $B$ is selected when $r_{A B}>\xi$.

2.5. CSP to Remove the Unimportant Reactions. In 2008, $\mathrm{Lu}$ and Law [21] developed the CSP method to remove the unimportant reactions through an importance index after eliminating the unimportant species. For this method, the importance index of the reaction is defined as

$$
I_{A, i}=\frac{\left|v_{A, i} \omega_{i}\right|}{\sum_{i=1, I}\left|v_{A, i} \omega_{i}\right|} .
$$

$i$ th reaction is considered not important for a reaction state when $I_{A, i}<\xi$.

2.6. Time-Scale Reduction with QSSA. QSSA assumes that the net production rate of the QSS species is zero to obtain less stiffness of the chemical reaction system. To apply the QSSA method, the QSS species need to be identified by CSP theory. CSP theory is based on the Jacobian matrix $\mathbf{J}$ in the following form:

$$
\mathbf{J}=\frac{d \mathbf{g}}{d \mathbf{y}},
$$

where $\mathbf{y}$ is the vector of the species concentration and $\mathbf{g}$ is the source term, including the contribution from chemical reactions. 


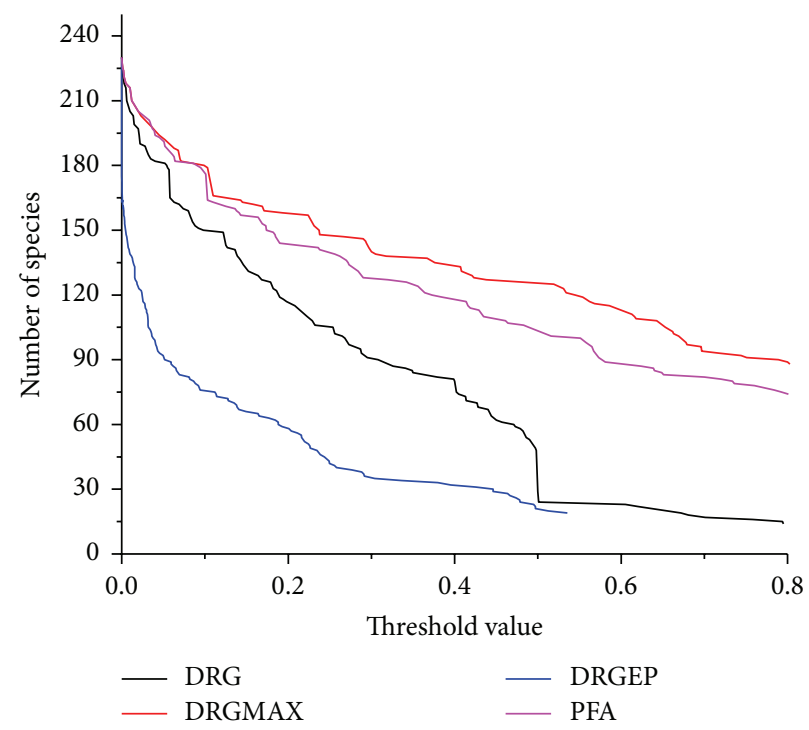

Figure 1: Number of species of the skeletal mechanisms generated as a function of the threshold values.



FIgURE 2: Maximum error of the predicted autoignition delay time of the skeletal mechanisms.

Once the fast and slow subspaces are decoupled, the Jacobian matrix $\mathbf{J}$ can become

$$
\begin{aligned}
\mathbf{J} & =\left(\begin{array}{ll}
\mathbf{A}_{\text {fast }} & \mathbf{A}_{\text {slow }}
\end{array}\right)\left(\begin{array}{cc}
\boldsymbol{\Lambda}_{\text {fast }} & 0 \\
0 & \boldsymbol{\Lambda}_{\text {slow }}
\end{array}\right)\left(\begin{array}{c}
\mathbf{B}_{\text {fast }} \\
\mathbf{B}_{\text {slow }}
\end{array}\right), \\
\mathbf{A} & =\left(\begin{array}{ll}
\mathbf{A}_{\text {fast }} & \mathbf{A}_{\text {slow }}
\end{array}\right), \\
\mathbf{B} & =\left(\begin{array}{c}
\mathbf{B}_{\text {fast }} \\
\mathbf{B}_{\text {slow }}
\end{array}\right),
\end{aligned}
$$

where $\mathbf{A}$ and $\mathbf{B}$ are the basis vectors and $\boldsymbol{\Lambda}$ is the diagonal matrix.

The criterion for the QSS species can be expressed as [22]

$$
\left|Q_{i}\right|<\varepsilon, \quad \mathbf{Q}=\mathbf{A}_{\text {slow }} \mathbf{B}_{\text {slow }},
$$

where $\mathbf{Q}$ is the projection matrix to the slow subspace and $\varepsilon$ is the threshold to identify the QSS species.

\section{Reduction Strategies}

3.1. Skeletal Reduction. The data sampled for reduction [2224] were from autoignition of the 230-species detailed mechanism under pressures from 1 to $45 \mathrm{~atm}$, equivalence ratios from 0.3 to 2 , and initial temperatures from $690 \mathrm{~K}$ to $1430 \mathrm{~K}$. The species $\mathrm{C}_{4} \mathrm{H}_{10}, \mathrm{~N}_{2}, \mathrm{O}_{2}, \mathrm{CO}_{2}$, and $\mathrm{H}_{2} \mathrm{O}$ were selected as the initial important species. The DRG, DRGEP, DRGMAX, and PFA methods were used to generate different skeletal mechanisms with different threshold values $\xi$. Figure 1 shows the relationship between the threshold values and the number of species from different reduction methods. Figure 2 shows 


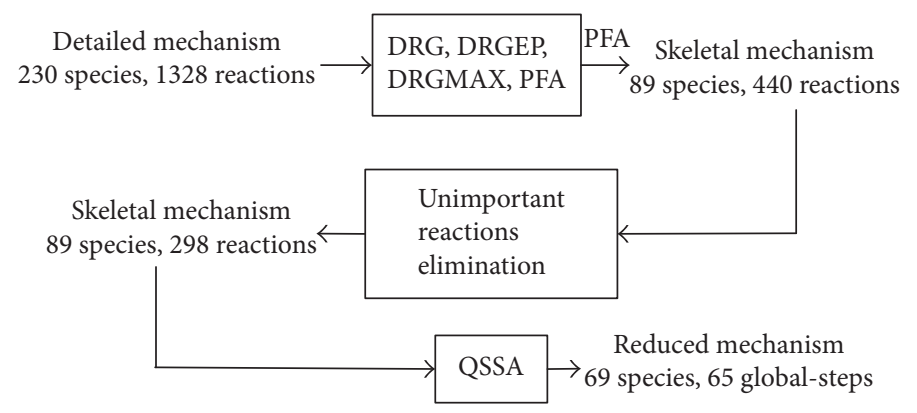

FIgURE 3: Flowchart of the reduction procedures for the $n$-butane mechanism.

the relationship between the number of species and the maximum error of the ignition delay time for the different skeletal mechanisms from different reduction methods. When the number of species of different reduction methods is similar, the maximum error of the ignition delay time is different. Figures 1 and 2 also show that we can generate the smallest skeletal mechanism consisting of 89 species and 440 elementary reactions by the PFA method. Compared with the detailed mechanism, the skeletal mechanism eliminated 141 species, and the maximum and averaged errors of the ignition delay time were $21.26 \%$ and $6.5 \%$.

We further removed the unimportant reactions of the 89 species and 440 elementary reactions of the skeletal mechanism. The data sampled and the important species selected were the same as for the PFA method. By comparing different thresholds, the final skeletal mechanism consisting of 89 species and 298 elementary reactions was derived with the requirement of $\xi=0.1916$. Compared with the 89 -species skeletal mechanism, the final skeletal mechanism eliminated 150 elementary reactions, and the maximum and the averaged errors of the ignition delay time were $22.37 \%$ and $8.9 \%$.

3.2. Time-Scale Reduction with QSSA. By using CSP to identify the QSS species, the 89-species skeletal mechanism can be further reduced. Again, using the databases from the autoignition of the 230-species detailed mechanism under pressures from 1 to $45 \mathrm{~atm}$, equivalence ratios from 0.3 to 2, and initial temperatures from $690 \mathrm{~K}$ to $1430 \mathrm{~K}$, the time scales of each species were calculated for each data point [25]. Choosing $\varepsilon=0.01538993$, species were identified as global QSS species, namely, C2H3O1-2, SC4H9O, HOCH2O, $\mathrm{CH} 3 \mathrm{CO} 2, \mathrm{C} 4 \mathrm{H} 8 \mathrm{OH}-2 \mathrm{O} 2$, $\mathrm{SC} 4 \mathrm{H} 8 \mathrm{OH}, \mathrm{CH} 3 \mathrm{CO}, \mathrm{C} 3 \mathrm{H} 2$, C3H6OOH1-2, C3H6OOH1-3, C4H8OOH2-4, C3H5O, $\mathrm{CH} 2(\mathrm{~S}), \mathrm{C} 4 \mathrm{H} 8 \mathrm{OOH} 1-3, \mathrm{C} 4 \mathrm{H} 8 \mathrm{OOH} 2-3, \mathrm{SC} 4 \mathrm{H} 9, \mathrm{HCO}$, $\mathrm{NC} 3 \mathrm{H} 7$, and PC4H9. Finally, we obtained a 69 -species and 65-global-step reduced mechanism.

As a summary, Figure 3 shows the flowchart of the reduction procedures and the sizes of the intermediate mechanisms. The final reduced mechanism by integration of different reduction approaches is approximately 4 times smaller than the detailed mechanism. The reduction by an individual method alone is less powerful. The error tolerances of the ignition delay time were smaller than $30 \%$ in the reduction steps.

\section{Reduction Validation}

The ignition delay time, as an important flame feature, can be calculated in the closed homogeneous constant-volume reactor. Figure 4 shows the calculated ignition delay time using the 230-species detail, the 89 -species skeletal, and the 69 -species reduced mechanisms under pressures from 1 to $45 \mathrm{~atm}$, equivalence ratios from 0.3 to 2 , and initial temperatures from $690 \mathrm{~K}$ to $1430 \mathrm{~K}$. The 89 -species skeletal and 69-species reduced mechanisms accurately mimic the 230 species detailed mechanism for the entire parameter range, exhibiting slightly larger deviations around the negative temperature coefficient (NTC) zone. The deviations are primarily induced by the relatively large temperature interval in the NTC zone, which eliminates some active species and elementary reactions.

Figure 5 shows the temperature profiles calculated in the closed homogeneous constant-volume reactor using these mechanisms for the pressure of $1 \mathrm{~atm}$ and equivalence ratio range of $0.3-1$. The temperature variation comparison is similar for the entire equivalence ratio range, with slightly larger error for low initial temperature $\left(T_{0}=800 \mathrm{~K}\right)$.

Figure 6 shows the computed mole fraction of the main species for the pressure of $10 \mathrm{~atm}$ and equivalence ratio of 1 . The main species mole fractions have very close agreement, and the 69-species reduced mechanism has smaller errors than the 89-species skeletal mechanism.

To further examine the performance of the reduced mechanisms, the 1D laminar flame speeds were calculated with the 230-species detailed, 89-species skeletal, and 69species reduced mechanisms. Figure 7 shows that the curves for the reduced mechanisms are similar to those of the detailed mechanism, with the worst-case difference being less than $4.5 \mathrm{~cm} / \mathrm{s}$ under the pressure of $10 \mathrm{~atm}$.

\section{Conclusions}

A suite of methods was integrated for the systematic reduction of large $n$-butane detailed mechanisms comprising 230 species and 1328 reactions. The integrated method consists of two major steps, namely, skeletal reduction and global reduction (time-scale reduction). The PFA has been applied to the generation of a skeletal mechanism with 89 species and 440 elementary reactions by comparing the different 

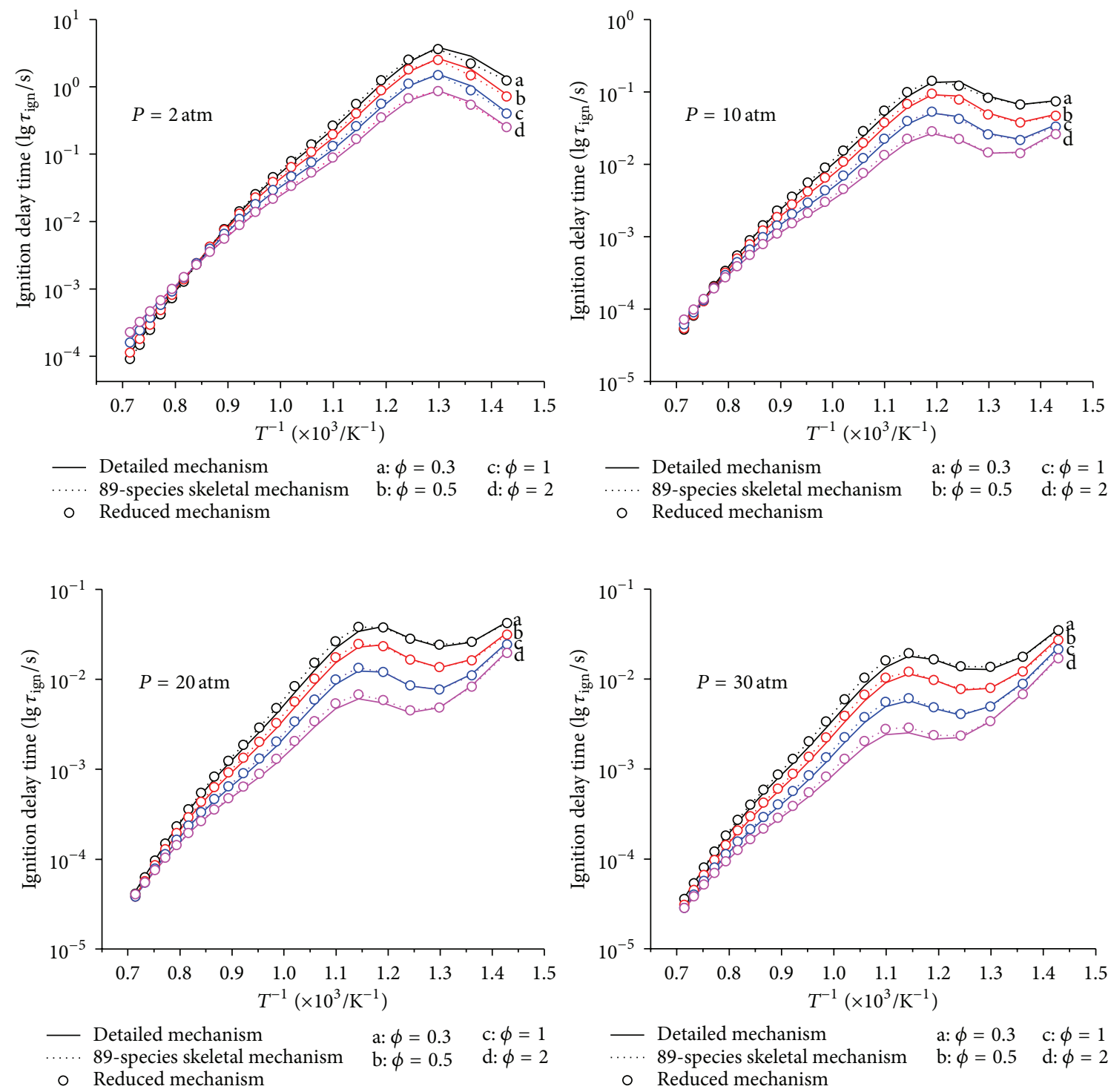

... 89-species

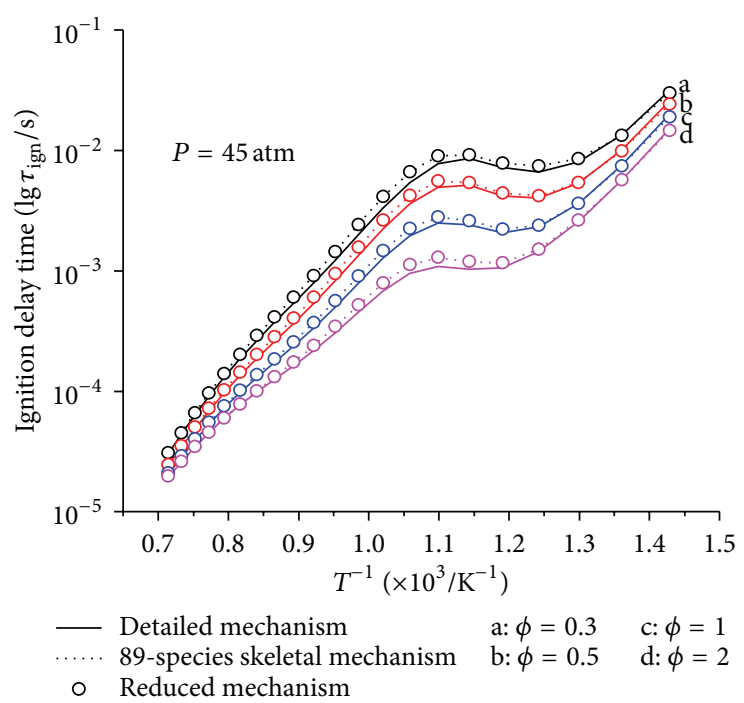

Figure 4: Ignition delay time of the $n$-butane/air mixture depended on the initial temperature between the 230-species detailed, 89 -species skeletal, and 69-species reduced mechanisms under different pressures and equivalence ratios. 

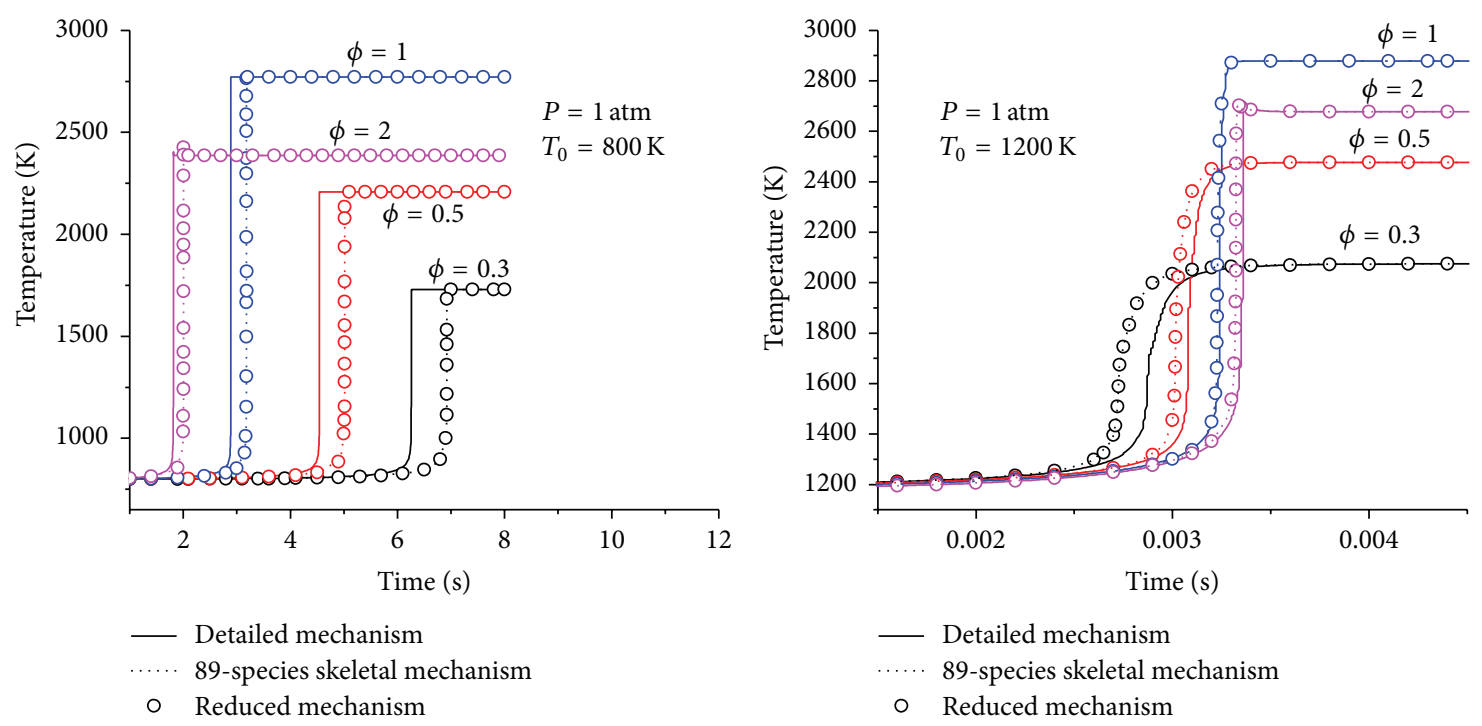

FIGURE 5: The temperature profiles of $n$-butane/air in constant-volume autoignition under the pressure of 1 atm, different initial temperatures, and different equivalence ratios, calculated with the 230-species detailed, 89-species skeletal, and 69-species reduced mechanisms.

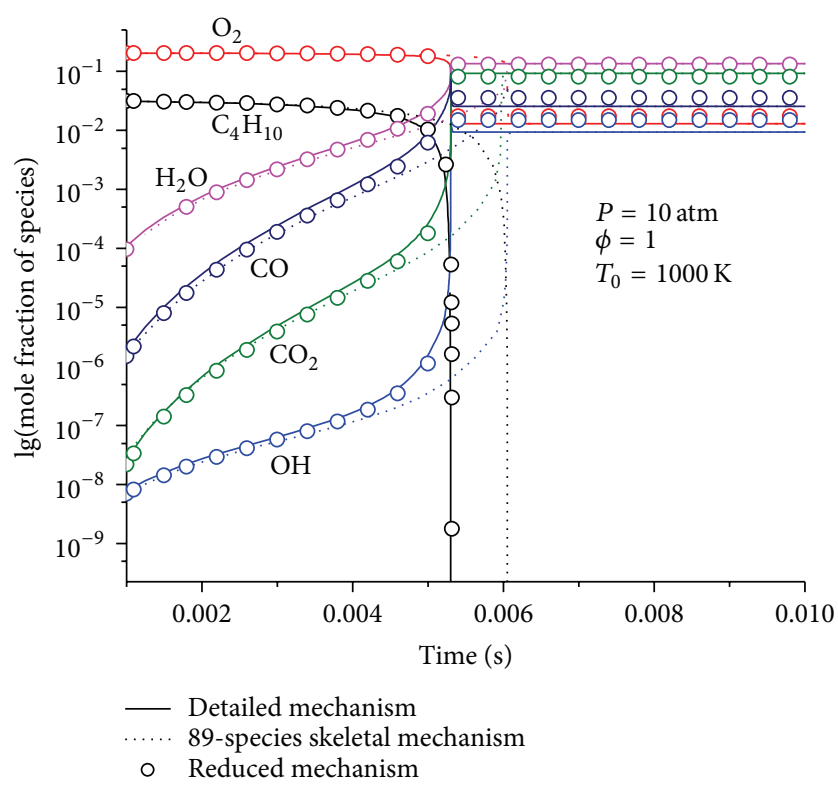

FIGURE 6: The mole fraction of $n$-butane/air for the main species in constant-volume autoignition under the pressure of $1 \mathrm{~atm}$, initial temperature of $T_{0}=1000 \mathrm{~K}$, and equivalence ratio of 1 , calculated with the 230-species detailed, 89-species skeletal, and 69-species reduced mechanisms.

methodologies based on directed relation graph reduction. The unimportant reactions were eliminated using the CSP importance index, resulting in an 89-species and 298elementary-reaction skeletal mechanism. By identifying the QSS species, the 89-species and 298-elementary-reaction skeletal mechanism can be simplified further to a reduced mechanism, including 69 species and 65-global-step reactions. High fidelity in the simulation was demonstrated.

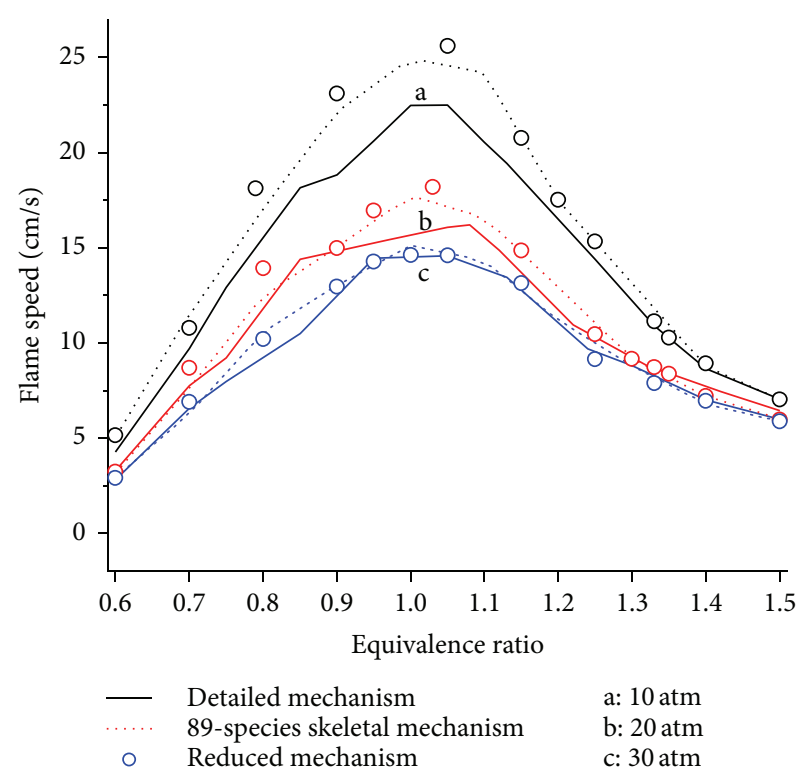

FIGURE 7: The dependence of the laminar flame speed on the equivalence ratio between the 230-species detailed, 89-species skeletal, and 69 -species reduced mechanisms at various pressures.

\section{Competing Interests}

The authors declare that they have no competing interests.

\section{Acknowledgments}

This work was supported by the National Natural Science Foundation of China (51006010) and the Scientific Research Foundation of Tianjin University of Technology and Education (RC14-13). 


\section{References}

[1] W. M. Yang, S. K. Chou, C. Shu, A. W. Li, and H. Xue, "Power generation at the micro scale," International Journal of Computational Engineering Science, vol. 4, no. 3, pp. 481-484, 2003.

[2] A. Mehra, X. Zhang, A. A. Ayón, I. A. Waitz, M. A. Schmidt, and C. M. Spadaccini, "Six-wafer combustion system for a silicon micro gas turbine engine," Journal of Microelectromechanical Systems, vol. 9, no. 4, pp. 517-527, 2000.

[3] S. J. Lee, A. Chang-Chien, S. W. Cha et al., "Design and fabrication of a micro fuel cell array with 'flip-flop' interconnection," Journal of Power Sources, vol. 112, no. 2, pp. 410-418, 2002.

[4] W. M. Yang, S. K. Chou, C. Shu, H. Xue, and Z. W. Li, "Development of a prototype micro-thermophotovoltaic power generator," Journal of Physics D: Applied Physics, vol. 37, no. 7, pp. 10171020, 2004.

[5] K. Maruta, "Micro and mesoscale combustion," Proceedings of the Combustion Institute, vol. 33, no. 1, pp. 125-150, 2011.

[6] S. Kojima, "Detailed modeling of n-butane autoignition chemistry," Combustion and Flame, vol. 99, no. 1, pp. 87-136, 1994.

[7] V. Warth, N. Stef, P. A. Glaude, F. Battin-Leclerc, G. Scacchi, and G. M. Côme, "Computer-aided derivation of gas-phase oxidation mechanisms: application to the modeling of the oxidation of n-butane," Combustion and Flame, vol. 114, no. 12, pp. 81-102, 1998.

[8] M. I. Strelkova, A. A. Safonov, L. P. Sukhanov et al., "Low temperature n-butane oxidation skeletal mechanism, based on multilevel approach," Combustion and Flame, vol. 157, no. 4, pp. 641652, 2010

[9] D. Healy, N. S. Donato, C. J. Aul et al., "n-Butane: ignition delay measurements at high pressure and detailed chemical kinetic simulations," Combustion and Flame, vol. 157, no. 8, pp. 15261539, 2010.

[10] T. F. Lu and C. K. Law, "A directed relation graph method for mechanism reduction," Proceedings of the Combustion Institute, vol. 30, no. 1, pp. 1333-1341, 2005.

[11] P. Pepiot-Desjardins and H. Pitsch, "An efficient error-propagation-based reduction method for large chemical kinetic mechanisms," Combustion and Flame, vol. 154, no. 1-2, pp. 67-81, 2008.

[12] W. Sun, Z. Chen, X. Gou, and Y. Ju, "A path flux analysis method for the reduction of detailed chemical kinetic mechanisms," Combustion and Flame, vol. 157, no. 7, pp. 1298-1307, 2010.

[13] Z. Y. Luo, T. F. Lu, M. J. MacIaszek, S. Som, and D. E. Longman, "A reduced mechanism for high-temperature oxidation of biodiesel surrogates," Energy and Fuels, vol. 24, no. 12, pp. 62836293, 2010.

[14] S. H. Lam, "Using CSP to understand complex chemical kinetics," Combustion Science and Technology, vol. 89, no. 5-6, pp. 375-404, 1993.

[15] M. Valorani, F. Creta, D. A. Goussis, J. C. Lee, and H. N. Najm, "An automatic procedure for the simplification of chemical kinetic mechanisms based on CSP," Combustion and Flame, vol. 146, no. 1-2, pp. 29-51, 2006.

[16] F. Wen and B. Zhong, "Skeletal mechanism generation based on eigenvalue analysis method," Acta Physico-Chemica Sinica, vol. 28, no. 6, pp. 1306-1312, 2012.

[17] H. Rabitz, M. A. Kramer, and D. Dacol, "Sensitivity analysis in chemical kinetics," Annual Review of Physical Chemistry, vol. 34, pp. 419-461, 1983.
[18] S. H. Lam and D. A. Gousiss, "The CSP method for simplifying kinetics," International Journal of Chemical Kinetics, vol. 26, no. 4, pp. 461-486, 1994.

[19] J. Y. Chen, "A general procedure for constructing reduced reaction mechanisms with given independent relations," Combustion Science and Technology, vol. 57, no. 1-3, pp. 89-94, 1988.

[20] U. Maas and S. B. Pope, "Simplifying chemical kinetics: intrinsic low-dimensional manifolds in composition space," Combustion and Flame, vol. 88, no. 3-4, pp. 239-264, 1992.

[21] T. F. Lu and C. K. Law, "Strategies for mechanism reduction for large hydrocarbons: $n$-heptane," Combustion and Flame, vol. 154, no. 1-2, pp. 153-163, 2008.

[22] T. F. Lu and C. K. Law, "A criterion based on computational singular perturbation for the identification of quasi steady state species: a reduced mechanism for methane oxidation with $\mathrm{NO}$ chemistry," Combustion and Flame, vol. 154, no. 4, pp. 761-774, 2008.

[23] S. H. Li, Y. M. Fang, F. Wang, P. Li, and X. Y. Li, "Systematic reduction and analysis of kinetic mechanism for hightemperature combustion of methyl heptanoate," Chemical Journal of Chinese Universities, vol. 34, no. 7, pp. 1714-1722, 2013.

[24] Y. M. Fang, Q. D. Wang, F. Wang, and X. Y. Li, "Reduction of the detailed kinetic mechanism for high-temperature combustion of n-dodecane," Acta Physico-Chimica Sinica, vol. 28, no. 11, pp. 2536-2542, 2012.

[25] S. H. Li, J. W. Liu, R. Li, F. Wang, N. X. Tan, and X. Y. Li, "Automatic chemistry mechanism reduction on hydrocarbon fuel combustion," Chemical Journal of Chinese Universities, vol. 36, no. 8, pp. 1576-1587, 2015. 

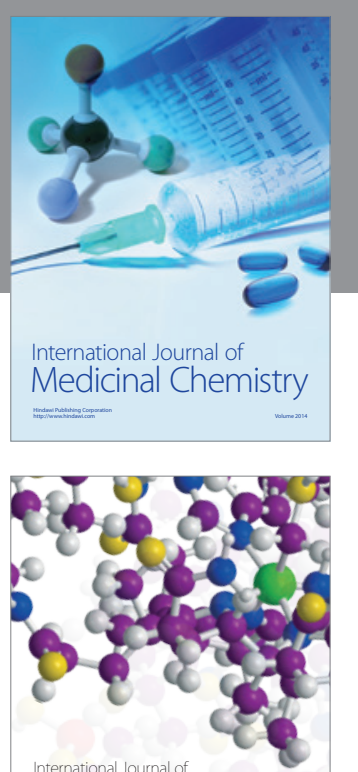

Carbohydrate Chemistry

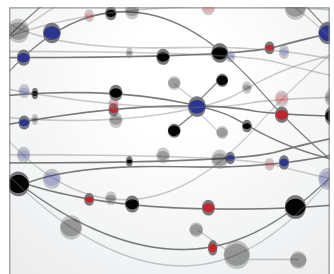

The Scientific World Journal
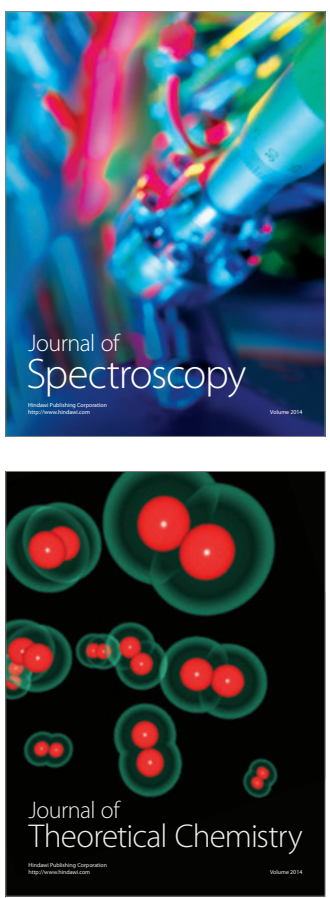
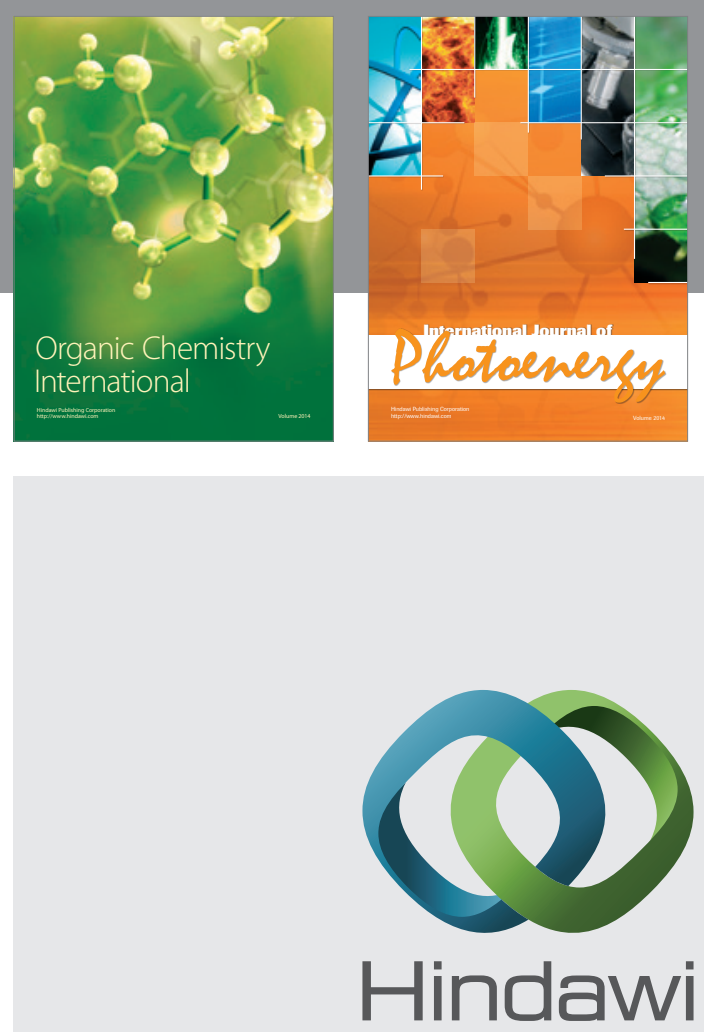

Submit your manuscripts at

http://www.hindawi.com

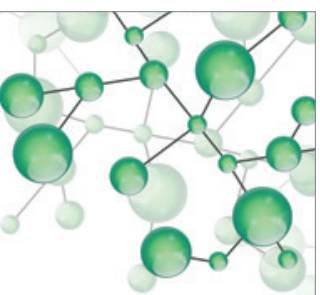

International Journal of

Inorganic Chemistry

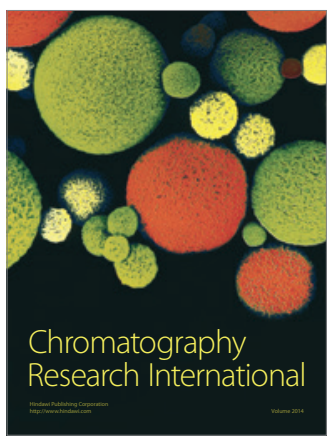

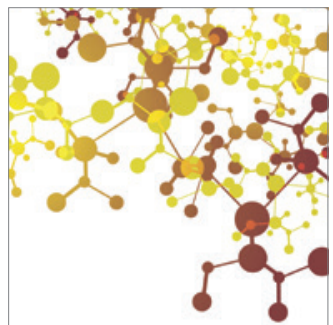

Applied Chemistry
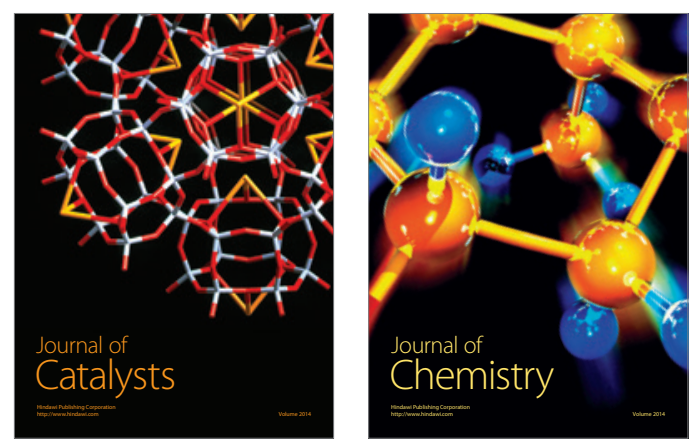
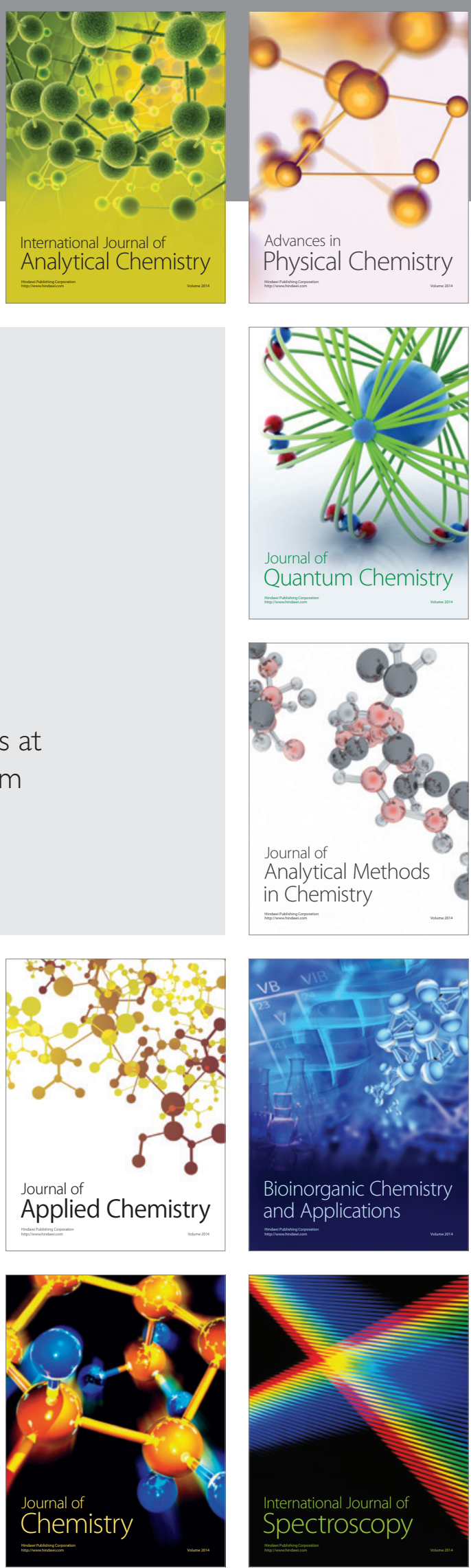